\title{
Doença de Parkinson: Revisão Clínica e Atualização
}

\section{Parkinson's Disease: Clinical Review and Update}

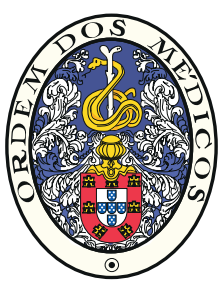

Verónica CABREIRA $\triangle^{1,2}$, João MASSANO ${ }^{1,2}$

Acta Med Port 2019 Oct;32(10):661-670 - https://doi.org/10.20344/amp.11978

\section{RESUMO}

A doença de Parkinson é a segunda doença neurodegenerativa mais comum, tendo sido documentado um aumento significativo da sua prevalência nas últimas três décadas. A fisiopatologia da doença assenta numa interação genética-ambiente, estimando-se que cerca de $5 \%-10 \%$ dos casos tenham causa genética monogénica. O diagnóstico é clínico, apoiado por investigação complementar adequada. Não dispomos ainda de uma forma de diagnosticar com certeza a doença de Parkinson in vivo, à exceção de testes genéticos em circunstâncias específicas, cuja utilidade é limitada a uma minoria de casos. Recentemente foram propostos novos critérios de diagnóstico, com o objetivo de melhorar a acuidade diagnóstica, com ênfase nas características que apontam para outras causas de parkinsonismo. As opções terapêuticas atualmente disponíveis são clinicamente úteis, pois têm a capacidade de melhorar os sintomas da doença e a qualidade de vida dos doentes. Após a introdução da levodopa, a estimulação cerebral profunda surgiu, mais recentemente, como a segunda intervenção terapêutica com importante impacto sintomático no tratamento desta doença. Os sintomas não motores e as complicações motoras são responsáveis por uma parte considerável da incapacidade na doença de Parkinson, pelo que devem ser identificados e tratados. A investigação científica atual foca-se na identificação de potenciais biomarcadores da doença, que permitam alcançar um diagnóstico certeiro e atempado, e na criação de terapêuticas mais eficazes, de modo a preencher as necessidades clínicas atualmente não satisfeitas. Este artigo apresenta uma revisão atualizada sobre a doença de Parkinson, guiando o leitor através dos conceitos mais atualizados nesta temática, de forma a permitir a sua aplicação na prática clínica diária.

Palavras-chave: Corpos de Lewy; Doença de Parkinson/diagnóstico; Doença de Parkinson/genética; Estimulação Cerebral Profunda; Levodopa

\section{ABSTRACT}

Parkinson's disease is the second most common neurodegenerative disorder, and a significant increase in its prevalence in the past three decades has been documented. Environmental and genetic factors contribute to the pathophysiology of this disease, and $5 \%-10 \%$ of cases have a monogenic cause. The diagnosis relies on clinical findings, supported by adequate testing. There is no absolute method to diagnose Parkinson's disease in vivo, except for genetic testing in specific circumstances, whose usefulness is limited to a minority of cases. New diagnostic criteria have been recently proposed with the aim of improving diagnostic accuracy, emphasizing findings that might point to other causes of parkinsonism. The available therapeutic options are clinically useful, as they improve the symptoms as well as the quality of life of patients. After the introduction of levodopa, deep brain stimulation emerged as the second therapy with an important symptomatic impact in the treatment of Parkinson's disease. Non-motor symptoms and motor complications are responsible for a large proportion of disability, so these should be identified and treated. Current scientific research is focused on the identification of disease biomarkers allowing correct and timely diagnosis, and on creating more effective therapies, thus fulfilling current clinical unmet needs. This paper presents an updated review on Parkinson's disease, guiding the readership through current concepts, and allowing their application to daily clinical practice.

Keywords: Deep Brain Stimulation; Levodopa; Lewy Bodies; Parkinson Disease/diagnosis; Parkinson Disease/genetics

\section{INTRODUÇÃo}

A doença de Parkinson (DP) é a segunda doença neurodegenerativa mais comum, superada apenas pela doença de Alzheimer. ${ }^{1}$ A sua descrição remonta a 1817 , quando James Parkinson descreveu os sintomas cardinais desta doença, mais tarde designada com o seu nome..$^{2,3}$ A DP integra o grupo das sinucleinopatias, caracterizadas pela acumulação da proteína alfa-sinucleína. Na DP esta agrega-se de forma anómala no tecido neuronal, originando os corpos de Lewy, característica patológica deste grupo de doenças. A acumulação de corpos de Lewy antecede os sinais neuro-imagiológicos de morte neuronal, ${ }^{4}$ correspondendo a um processo de neurodegenerescência que evolui lentamente e se propaga a diferentes áreas do sistema nervoso, nomeadamente à substantia nigra pars compacta, com consequente perda de neurónios dopaminérgicos e respetivos sintomas motores da doença, que se correlacionam com a extensão da neurodegenerescência. ${ }^{5,6} \mathrm{~A}$ epidemiologia da doença varia consoante a localização geográfica e a metodologia dos estudos. Na Europa estima-se que se situe nos 257 a 1400 casos por 100 mil habitantes. Um recente estudo em Portugal, transversal, com uma amostra populacional acima dos 50 anos de idade, calculou a prevalência da DP em 180/100 000 habitantes (IC 95\% 30 - 327/100 000), admitindo-se uma provável subestimativa. ${ }^{7}$ A DP surge geralmente entre os 50 e os 80 anos de idade, com um pico na sétima década de vida, sendo mais prevalente nos homens (3:2). ${ }^{1}$ Este padrão poderá refletir a importância de certos fatores ambientais e sociais na DP e a sua interação com outras variáveis, como genes, níveis hormonais, efeitos da gravidez e diferentes profissões ou exposições ambientais. ${ }^{8}$ Nas últimas décadas, a prevalência da DP tem vindo a aumentar, sobretudo nos países mais desenvolvidos: dados do Global Burden of

\footnotetext{
1. Serviço de Neurologia. Centro Hospitalar Universitário de São João. Porto. Portugal.

2. Departamento de Neurociências Clínicas e Saúde Mental. Faculdade de Medicina. Universidade do Porto. Porto. Portugal.

$\square$ Autor correspondente: João Massano. jmassano@med.up.pt
}

Recebido: 23 de fevereiro de 2019 - Aceite: 05 de abril de 2019 | Copyright $\odot$ Ordem dos Médicos 2019 
Diseases, Injuries, and Risk Factors Study 2016 estimam a existência de cerca de 6,1 milhões de pessoas diagnosticadas com DP em todo o mundo, valor que não ultrapassava os 2,5 milhões em $1990 .^{9}$ Este aumento não parece ser explicado apenas pelo envelhecimento da população, existindo outros fatores como um melhor reconhecimento da doença e fatores ambientais ou sociais. ${ }^{10}$ A sobrevivência média dos doentes tem vindo a aumentar desde os 9,4 anos na era pré-levodopa (o primeiro marco histórico no tratamento da DP), para 13,1 anos em 1993, na era pré-estimulação cerebral profunda (segundo marco histórico no tratamento da DP), situando-se atualmente nos 14,6 $( \pm 7,7)$ anos, comparativamente aos 23,3 anos estimados para um adulto de 60 anos da população geral. Dada a complexidade e heterogeneidade da DP, é difícil estimar o prognóstico de um doente individual. A idade mais avançada, o género masculino, co-morbilidades (e.g. fatores de risco vascular, cancro, síndrome metabólica, baixo índice de massa corporal), o atingimento motor axial, a deterioração cognitiva e disfunção autonómica precoces têm sido apontados como fatores preditores independentes de pior prognóstico. ${ }^{11-16}$ Recentemente, níveis baixos de vitamina B12 foram associados a pior prognóstico ${ }^{17}$. Este texto pretende ser um guia para os clínicos que habitualmente contactam com doentes com DP, com especial ênfase nas características clínicas da doença, critérios de diagnóstico atuais e as opções contemporâneas para o tratamento da doença.

\section{MATERIAL E MÉTODOS}

A revisão bibliográfica resultou da pesquisa de artigos publicados na Pubmed/Medline (palavras-chave: 'Parkinson Disease'; 'Parkinson Disease, Secondary'; 'Parkinsonian Disorders'; 'motor complications'; 'biomarker'; 'Lewy bodies'; 'genetics'; 'therapy'; 'deep brain stimulation') e de informação iconográfica/guidelines da International Parkinson and Movement Disorder Society (MDS). Desta pesquisa e posterior seleção dos artigos, realizada em função da sua pertinência e atualidade, resultaram artigos publicados, em língua inglesa ou portuguesa, entre 1988 e 2018.

\section{RESULTADOS \\ Etiopatogénese}

Diversos fatores apontam para uma etiopatogénese heterogénea, alicerçada na interação genética-ambiente. A evidência sugere que o traumatismo craniano, a exposição a pesticidas agrícolas e a outras toxinas como o manganésio, aumentam o risco futuro de DP esporádica. ${ }^{1,8}$ Mais recentemente, a inflamação intestinal tem sido apontada como eventual fator desencadeante da doença. ${ }^{18}$ Contrariamente, a evidência sobre fatores protetores é mais escassa e assente sobretudo em estudos de casocontrolo, sugerindo que níveis mais elevados de ácido úrico sérico, consumo de café e de tabaco se associam a menor risco de $\mathrm{DP}^{19}$.

Embora a maioria dos casos seja esporádica, cerca de $55 \%-10 \%$ dos casos de DP têm causa monogénica e cerca de $20 \%$ dos pacientes com DP reportam pelo menos um familiar em primeiro ou segundo grau afetado pela doença. ${ }^{20}$ Com o aumento da relação custo-eficiência dos testes genéticos e a disponibilização de novas técnicas como next generation sequencing, a tendência para a identificação de loci patogénicos é crescente e têm sido descritas várias formas monogénicas da doença, incluindo autossómicas dominantes (e.g. LRRK2, SNCA, VPS35, EIF4G1, CHCHD2...), autossómicas recessivas (e.g. PRKN, PINK1, $D J-1)$ e ligadas ao $X(R A B 39 B) \cdot{ }^{21}$ A mutação genética associada a DP mais frequentemente encontrada na população ocorre no gene da glucocerebrosidase (GBA). Uma cópia alterada deste gene foi pela primeira vez associada a maior risco de DP em 2004; no entanto, a penetrância genética, embora variável, é globalmente reduzida na população geral. ${ }^{20} \mathrm{O}$ mecanismo pelo qual as mutações do gene $G B A$ aumentam o risco de DP envolve possivelmente modelos de ganho de função que promovem a agregação de alfa-sinucleína, embora esta relação permaneça ainda por esclarecer. Nestes doentes, o fenótipo motor é semelhante à forma esporádica da doença, embora se verifique um maior risco de disfunção cognitiva. Esta mutação é mais frequente em populações descendentes de judeus Ashkenazi. ${ }^{22,23}$ Uma revisão recente de Ferreira e Massano ${ }^{21}$ aborda as principais alterações genéticas causadoras de DP e suas correlações clínico-patológicas.

\section{Diagnóstico clínico}

Atualmente não existe um biomarcador ou teste que nos permita afirmar categoricamente o diagnóstico da doença in vivo, à exceção de testes genéticos específicos, úteis apenas numa minoria de casos. ${ }^{21} \mathrm{~A}$ deteção de alfa-sinucleína na retina, pele, urina, plasma e LCR tem sido estudada como potencial biomarcador da doença, mas os resultados até à data não permitem a sua aplicação na prática clínica. ${ }^{24,25} \mathrm{O}$ diagnóstico baseia-se portanto fundamentalmente nos sintomas clínicos e inicia-se com a confirmação da existência de parkinsonismo, uma designação sindrómica caracterizada por um conjunto de sintomas motores (dos quais a bradicinésia é a característica fundamental) que podem ser observados em múltiplas doenças. ${ }^{19,26}$ Pelo exposto se compreende que nem o tremor de repouso nem a rigidez são sintomas obrigatórios por si só para o diagnóstico e, na ausência de bradicinésia, não é possível fazer o diagnóstico clínico de DP. A DP é a causa mais frequente de parkinsonismo neurodegenerativo. ${ }^{1}$

Seguidamente à história clínica e exame físico detaIhados, pode ser necessária investigação adicional para exclusão de outras causas de parkinsonismo. O diagnóstico clínico é suportado pela resposta favorável à terapêtica dopaminérgica, sobretudo a levodopa. Na tentativa de aglomerar os sintomas-chave da DP e os fatores de exclusão favoráveis a outros diagnósticos alternativos surgiram em 1988 os critérios clínico-patológicos do UK Brain Bank, ${ }^{27}$ ainda hoje amplamente adotados na seleção de doentes para estudos observacionais e aleatorizados, e também na prática clínica, apesar da sua sensibilidade para um 
diagnóstico precoce rondar os $80 \%$, sendo necessária observação prospectiva dos doentes para detetar sintomas que permitam ter em consideração diagnósticos alternativos. De referir que um dos critérios de exclusão, outrora utilizado ("mais do que um familiar afectado"), não é mais tido em conta no atual processo de diagnóstico, pelas razões já referidas. De facto, apesar das várias limitações, a análise histológica post-mortem é ainda hoje o padrão utilizado para validar novos critérios e analisar a sua aplicabilidade. Com o reconhecimento das novas formas genéticas da doença, surgiu um novo fenótipo 'clinico-genético' da doença: doentes com mutações genéticas (e.g. PRKN) sem deposição tecidular de alfa-sinucleína. ${ }^{28}$ Recentemente, a MDS sugeriu novos critérios com o objetivo de melhorar a fluidez e acuidade diagnóstica desde a primeira consulta, salientando-se o realce dos sintomas não motores. ${ }^{28}$ $\mathrm{Na}$ Fig. 1 propomos um algoritmo de diagnóstico de DP com base nestes novos critérios, recomendando-se em particular uma análise detalhada dos critérios de exclusão e das red flags para o diagnóstico de DP.

É possível demonstrar a existência de degenerescência nigroestriada in vivo através de imagiologia nuclear usando, por exemplo, a tomografia computadorizada de emissão única de fotões (SPECT) com ${ }^{123}$ |-ioflupano. Este método é sensível para demonstrar degenerescência neuronal dopaminérgica pré-sináptica no estriado, sem contudo identificar a sua etiologia específica (DP versus outras causas de parkinsonismo degenerativo). ${ }^{29-31}$ Mais recentemente descobriu-se que, paralelamente à degenerescência nigroestriada, há uma desnervação simpática cardíaca na DP e a cintigrafia miocárdica com ${ }^{123}$ |-MIBG (metaiodobenzilguanidina) aumenta a especificidade diagnóstica. ${ }^{28,32}$ Dado o custo elevado destes exames e a necessidade de uma interpretação cuidadosa, estes devem ser requisitados apenas quando existe dúvida diagnóstica razoável e/ou previsão de modificação na gestão clínica, e de preferência por Neurologistas familiarizados com os mesmos. $\mathrm{Na}$ tentativa de identificar biomarcadores da doença de forma não invasiva, a ultrassonografia transcraniana tem sido estudada como um método potencial de avaliação da degerescência nigroestriada, que avalia a hiperecogenicidade característica da substantia nigra na DP. No entanto, tem duas limitações principais: dependência importante do operador e da qualidade da janela óssea temporal do doente; a sua utilização, apesar de contemplada em recomendações europeias de 2013, encontra-se ainda limitada a contextos de investigação. ${ }^{33,34}$

Os exames de neuroimagem estrutural cerebral como a tomografia computadorizada e a ressonância magnética (RM) cerebral permitem excluir lesões estruturais de parkinsonismo, por exemplo doença vascular cerebral ou tumores intracranianos. ${ }^{35}$ A visualização em RM cerebral do 'sinal do colibri' na paralisia supranuclear progressiva (PSP), o 'sinal da cruz' na atrofia de múltiplos sistemas (AMS), ou a hiperintensidade dos pedúnculos cerebelosos médios na síndrome de ataxia e tremor ligada ao X-frágil (FXTAS) sugerem outras causas específicas de parkinsonismo. ${ }^{36,37}$
Também é importante solicitar estudo analítico adequado no sentido de excluir alterações que podem causar parkinsonismo, tremor, ${ }^{38}$ ou lentificação que simule parkinsonismo, nomeadamente anemia, disfunção tiroideia, alterações hidro-electrolíticas, doenças renal ou hepática crónicas, sífilis e infeção VIH.

\section{Sintomas motores}

- Bradicinésia - diminuição progressiva da velocidade e amplitude dos movimentos alternados e repetidos, executados o mais rápido e amplamente possível (e.g. abrir e fechar a mão, oponência do polegar e indicador, pronação-supinação das mãos, bater repetidamente com o calcanhar no chão). O movimento corporal espontâneo também está diminuído (bradicinésia global). A hipomimia (face inexpressiva ou imóvel, diminuição do pestanejo, lábios afastados), a hipofonia (voz com menor volume) e a micrografia (caligrafia mais pequena, por vezes imperceptível) são manifestações de bradicinésia. No extremo, pode existir interrupção completa do movimento (e.g. bloqueio ou freezing). É fundamental não confundir bradicinésia com lentidão simples, que pode ser causado por diminuição da força muscular, espasticidade, dor, fadiga geral (ex. anemia), hipotiroidismo, encefalopatias metabólicas ou tóxicas, sedação ou sintomas depressivos. Desta forma evitam-se erros de diagnóstico e podem identificar-se causas potencialmente tratáveis.

- Rigidez - aumento do tónus muscular, com resistência na movimentação passiva de um segmento ao longo de todo o movimento ('em cano de chumbo'). Testa-se avaliando, por exemplo, a flexão e a extensão cervical, do cotovelo, punho e articulação do joelho. Não é alterada pela velocidade da movimentação (ao contrário da espasticidade causada por lesão piramidal) e aumenta com manobras de ativação como a movimentação voluntária simultânea de outro membro - manobra de Froment. A rigidez 'em roda dentada' resulta da sobreposição do tremor e da rigidez, sendo mais facilmente detetável na articulação do punho.

- Tremor de repouso - movimento involuntário, rítmico e oscilatório, de frequência $4-6 \mathrm{~Hz}$. Avalia-se pela observação dos membros relaxados e apoiados numa superfície, sem ação da gravidade (ex. braços da cadeira). A fim de realçar o tremor pede-se ao doente para encerrar os olhos e executar uma tarefa cognitiva (e.g. contagem numérica decrescente). Na DP, o mais típico é observarse um tremor com fricção repetida do polegar e indicador, que visualmente se assemelha ao ato de contar moedas (ou pill-rolling). O tremor pode surgir noutras regiões, como os membros inferiores, a língua e a mandíbula. O tremor isolado da cabeça é raro na DP e deve alertar para um diagnóstico alternativo, nomeadamente tremores não degenerativos (ex. essencial, distónico, iatrogénico).

- Alterações posturais e da marcha - na DP a postura é tipicamente fletida e pode variar de alterações ligeiras igualmente presentes em pessoas idosas sem patologia, 


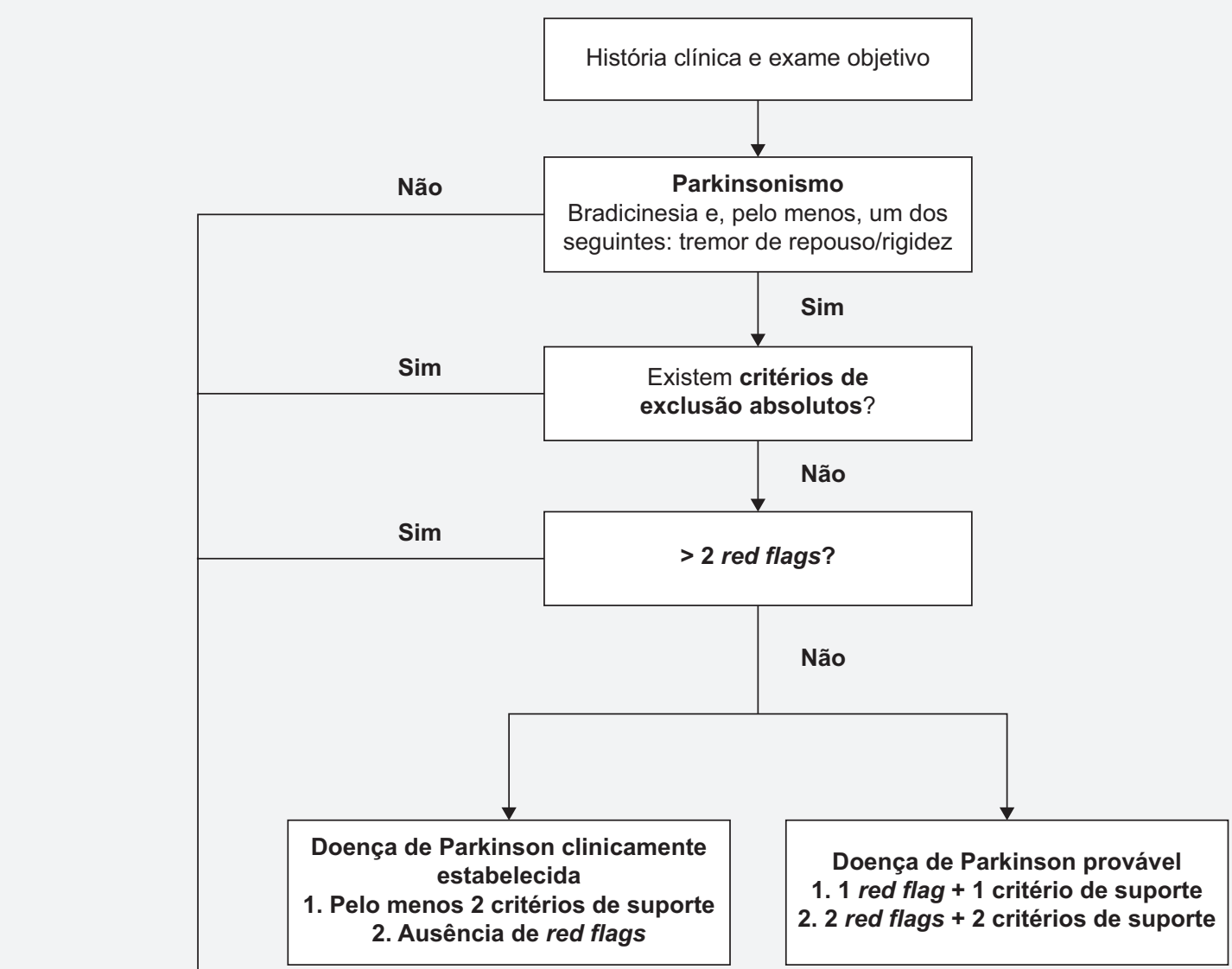

\section{Critérios de exclusão absolutos:}

- Sinais inequívocos de atingimento cerebeloso (e.g. ataxia da marcha ou de um membro, sacadas hipermétricas ...)

- Alterações dos movimentos oculares (lentificação das sacadas ou paralisia supranuclear)

- Demência frontotemporal variante comportamental ou afasia primária progressiva (primeiros 5 anos)

- Sintomas limitados exclusivamente aos membros inferiores durante, pelo menos, 3 anos

- História de tratamento farmacológico com algum antidopaminérgico (e.g. antipsicóticos) numa relação temporal e dose consistentes com parkinsonismo iatrogénico

- Ausência de resposta a altas dose de levodopa, apesar de gravidade da doença ser pelo menos moderada

- Alterações sensitivas corticais (agrafestesia, astereognosia), apraxia ou alien $\operatorname{limb}$

- Imagem nuclear de transportadores dopaminérgicos présinápticos (ex. SPECT com 123 I-ioflupano) sem alterações*

- Existência de um diagnóstico alternativo responsável pelos sintomas
Red flags para o diagnóstico de Doença de Parkinson:

- Progressão rápida da incapacidade para a marcha,

necessitando cadeira de rodas em 5 anos após início dos sintomas

- Ausência de progressão de sinais e sintomas ao longo de 5 ou mais anos de doença, exceto se estabilidade devida ao tratamento

- Envolvimento bulbar precoce (disfagia, disartria ou disfonia graves)

- Disfunção autonómica grave nos primeiros 5 anos de doença (e.g. hipotensão ortostática, incontinência ou retenção urinária graves)

- Estridor inspiratório ou suspiros inspiratórios frequentes

- Quedas recorrentes (> //ano) nos primeiros 3 anos

- Anterocollis desproporcionado

- Ausência de sintomas não motores apesar de sintomas motores há, pelo menos, 5 anos

- Sinais piramidais inexplicáveis

- Parkinsonismo simétrico bilateral desde o início

Critérios de suporte:

- Resposta clara a terapêutica dopaminérgica

- Discinésias induzidas pela levodopa

- Tremor de repouso num membro, documentado no exame físico

- Um destes é positivo

- Hipósmia inequívoca

- Desnervação cardíaca simpática na cintigrafia MIBG

Figura 1 - Diagnóstico de doença de Parkinson baseado nos critérios clínicos de diagnóstico da International Parkinson and Movement Disorder Society propostos em 2015. ${ }^{28}$ Estes substituem os antigos UK Parkinson's Disease Society Brain Bank Clinical Criteria ${ }^{27}$ criados há cerca de 25 anos, antes da identificação da alfa-sinucleína como característica patológica central da doença, do reconhecimento dos sintomas não motores e da identificação de formas genéticas da DP. A introdução de dados de exames complementares de diagnóstico reflete um melhor conhecimento da fisiopatologia da doença e os avanços tecnológicos ocorridos, entretanto.

*A introdução deste novo critério de exclusão não deve ser interpretada como a indicação para realização deste exame, mas antes como uma ferramenta útil a requerer numa minoria dos doentes (vide texto para detalhes). 
até uma postura mais acentuada com flexão pronunciada do tronco, a qual tem a particularidade de retificar em decúbito, distinguindo-se assim da cifose dorsal de causa osteoarticular. Pode observar-se durante a marcha e no pull test, em que o examinador se coloca atrás do doente e aplica um impulso para trás nos ombros deste, com o objetivo de avaliar a capacidade do doente contrariar o desequilíbrio imposto. Na DP a marcha é lenta, de base estreita, com passos curtos e baixos, e há um aumento do tempo em duplo suporte dos membros inferiores $^{39}$. Tipicamente há redução assimétrica do normal balanceio dos membros superiores durante a marcha; as voltas podem ser decompostas com múltiplos pequenos passos. Mais raramente surge festinação (sucessão muito rápida de passos dando a ilusão que o doente corre constantemente atrás do próprio centro de gravidade). Os bloqueios da marcha (freezing of gait) podem ser espontâneos, mas para os desencadear pode ser necessário solicitar aos doentes que atravessem espaços mais estreitos como as portas.

A existência de sintomas simétricos, quedas ou instabilidade postural numa fase precoce, sinais cerebelosos e disautonomia severa [mais indicativos de atrofia de múltiplos sistemas (AMS)] e alterações patológicas nos movimentos oculares [e.g. paralisia supranuclear do olhar vertical na paralisia supranuclear progressiva (PSP)] devem ser interpretadas como red flag diagnósticas, entre outras (Fig. 1). ${ }^{26,28}$ Aos doentes que se apresentam com os primeiros sintomas antes dos 40 anos de idade, atribui-se a designação de parkinsonismo juvenil, que é causado muitas vezes por mutações autossómicas recessivas, nomeadamente do gene PRKN (que codifica a proteína parkin). Nestes doentes é frequente observar hiperreflexia ósteotendinosa, distonia precoce e progressão motora lenta, mas maior propensão para flutuações motoras ${ }^{40}$.

\section{Sintomas não motores}

A DP é hoje reconhecida como uma doença multissistémica do sistema nervoso. Além dos sintomas motores cardinais, sabemos que a DP se caracteriza por uma panóplia de sintomas não motores, cuja identificação permite melhorar os cuidados clínicos prestados, monitorizar a progressão da doença e melhorar a nossa compreensão acerca da sua evolução ${ }^{19,41}$.

São exemplos de sintomas não motores as manifestações neuropsiquiátricas da doença como deterioração cognitiva, depressão, ansiedade, psicose, apatia e fadiga; queixas gastrointestinais como disfagia, enfartamento e obstipação; autonómicas como retenção ou urgência urinárias, disfunção sexual, sialorreia, hipersudorese, hipotensão ortostática; manifestações sensitivas como hiposmia e dor; manifestações visuais como alterações da perceção do contraste, ilusões e alucinações visuais (que podem ser complexas e bem formadas, como pessoas); distúrbios do sono com sonhos vívidos e atividade hipermotora noturna na perturbação do comportamento do sono REM (rapid eye movement), hipersonolência diurna e síndrome das pernas inquietas; entre outros. ${ }^{41,50}$ A deterioração cognitiva (continuum entre défice cognitivo ligeiro e demência) caracteriza-se por disfunção da atenção, memória de trabalho, funções executivas e eventualmente da linguagem, memória episódica e funções visuo-espaciais. A demência associada à DP pode afetar $80 \%$ dos doentes a longo prazo. ${ }^{42,43}$ Na prática clínica pode ser difícil obter avaliação neuropsicológica detalhada, pelo que diversas escalas de rastreio cognitivo foram validadas especificamente em populações com DP, sendo o teste Montreal Cognitive Assessment (MoCA) aquele que se encontra mais amplamente validado e que pode ser aplicado em cerca de 15 - 20 minutos. ${ }^{44}$ Este teste deve ser aplicado por profissionais com treino mínimo na sua execução e interpretação. Enquanto a demência surge tipicamente numa fase mais avançada da DP, outros sintomas, nomeadamente a perturbação do comportamento do sono REM e a hiposmia podem aparecer anos ou décadas antes dos sintomas motores (fase prodrómica ou 'pré-motora' da doença). ${ }^{6}$ Os sintomas nãomotores são frequentemente o primeiro sintoma que motiva a procura pelos serviços médicos, pelo impacto que condicionam na qualidade de vida dos doentes, ${ }^{45}$ mas são inespecíficos e podem surgir em pessoas sem DP, pelo que não deve ser levantada a hipótese deste diagnóstico nas pessoas que não tenham os sintomas motores típicos da doença. A Tabela 1 resume os principais sintomas não motores e intervenções terapêuticas recomendadas.

À semelhança dos sintomas motores, a progressão da doença cursa com flutuações nos sintomas não motores, que podem ser independentes das flutuações motoras e afetar de forma importante a qualidade de vida dos doentes. Há várias categorias de flutuações, nomeadamente autonómica, sensitiva/dolorosa e neuropsiquiátrica (e.g. apatia, depressão, ansiedade e lentificação cognitiva a predominarem nos períodos de 'off e perturbações do controlo dos impulsos mais comuns nos períodos de 'on'). Embora os mecanismos que estão na base destas flutuações permaneçam ainda por esclarecer, importa rastreá-las dadas as implicações terapêuticas; a evidência recente sugere benefício da cirurgia de estimulação cerebral profunda [deep brain stimulation, (DBS)] nestas flutuações, tal como nas flutuações motoras. ${ }^{46}$

Atualmente não se justifica o rastreio de sintomas não motores em indivíduos sem sintomas motores de parkinsonismo na tentativa de alcançar um diagnóstico ultra-precoce, pelo facto de não dispormos ainda de uma terapêutica com capacidade de atrasar a progressão da DP. Mesmo os familiares em primeiro grau de doentes com DP, alguns deles até com mutações dos genes $L R R K 2$ e $G B A$, não apresentam maior prevalência destes sintomas comparativamente à população geral, ${ }^{47}$ que justifiquem o rastreio de $\mathrm{DP}$, mesmo que haja legítima preocupação quanto ao risco futuro de desenvolver a doença.

\section{Diagnósticos diferenciais}

Dada a complexidade das manifestações clínicas e dos desafios que este diagnóstico nos coloca, a taxa de falsos 
Tabela 1 - Principais sintomas não motores e respetiva abordagem terapêutica

\begin{tabular}{|c|c|}
\hline Sintoma/síndrome & Intervenções sugeridas \\
\hline Demência & $\begin{array}{l}\text { - Rastrear e tratar causas de deterioração cognitiva (anemia, alterações hidro-electrolíticas, } \\
\text { disfunção tiroideia, défice de vitamina B12 ou ácido fólico, sífilis, infeção VIH) } \\
\text { - Inibidores da acetilcolinesterase (donepezilo, rivastigmina, galantamina), ou memantina } \\
\text { - Descontinuar medicamentos com efeitos deletérios sobre a cognição (incluindo anticolinérgicos, } \\
\text { benzodiazepinas, hidroxizina) e outras medicações não absolutamente necessárias }\end{array}$ \\
\hline $\begin{array}{l}\text { Alucinações, delírios, } \\
\text { confusão }\end{array}$ & $\begin{array}{l}\text { - Rastrear e tratar condições precipitantes (ex. infeções, alterações hidro-electrolíticas e metabólicas, } \\
\text { medicações de novo) } \\
\text { - Descontinuar terapêuticas não indispensáveis e/ou potencialmente iatrogénicas (incluindo } \\
\text { anticolinérgicos, benzodiazepinas, hidroxizina) } \\
\text { - Considerar redução da terapêutica anti-parkinsónica } \\
\text { - Considerar referenciação para Oftalmologia (rastreio e tratamento de causas de hipovisão, } \\
\text { como cataratas e retinopatia diabética) e Otorrinolaringologia (rastreio e tratamento de causas } \\
\text { de hipoacusia) } \\
\text { - Anticolinesterásicos possivelmente úteis (maior evidência para a rivastigmina) nas alucinações } \\
\text { - Para sintomas psicóticos considerar quetiapina (dose inicial } 25 \text { mg à noite); em casos refratários } \\
\text { e graves clozapina (começar com } 25 \text { mg, fazer monitorização semanal de hemograma por risco } \\
\text { de agranulocitose) } \\
\text { - Evitar antipsicóticos típicos (ex. haloperidol, cloropromazina) e quase todos os de segunda geração } \\
\text { (ex. risperidona, olanzapina) }\end{array}$ \\
\hline $\begin{array}{l}\text { Depressão e perturbações } \\
\text { da ansiedade }\end{array}$ & $\begin{array}{l}\text { Terapêuticas de primeira linha: SSRIs (ex. sertralina, escitalopram) e SNRIs (ex. venlafaxina, } \\
\text { duloxetina) } \\
\text { - Ciclo curto de benzodiazepinas (<4 semanas), para alívio da ansiedade } \\
\text { Otimizar terapêutica dopaminérgica (na depressão considerar adicionar ou aumentar a dose } \\
\text { de pramipexole) }\end{array}$ \\
\hline Hipotensão ortostática & $\begin{array}{l}\text { - Medidas gerais: uso de meias de compressão; evitar refeições de grande volume, álcool, ambientes } \\
\text { quentes, depleção de volume, diuréticos; rever fármacos anti-hipertensores, antidepressivos } \\
\text { tricíclicos, nitratos e bloqueadores alfa-adrenérgicos usados no tratamento da hipertrofia prostática } \\
\text { - Terapêutica farmacológica: midodrina }(2,5-10 \mathrm{mg} 3 / \text { dia), fludrocortisona }(0,05-0,1 \mathrm{mg} 1-2 / \text { dia }) \\
\text { - Diminuição da terapêutica dopaminérgica }\end{array}$ \\
\hline Sonolência diurna & $\begin{array}{l}\text { - Identificar e corrigir problemas do sono (incluindo síndrome de apneia obstrutiva do sono), tratar a } \\
\text { acinésia noturna e causas de noctúria } \\
\text { - Reduzir/descontinuar agonistas dopaminérgicos } \\
\text { - Adicionar modafinil ou considerar metilfenidato }\end{array}$ \\
\hline $\begin{array}{l}\text { Perturbação do } \\
\text { comportamento do sono } \\
\text { REM }\end{array}$ & $\begin{array}{l}\text { - Clonazepam }(0,5-2 \mathrm{mg}) \text { ao deitar } \\
\text { - Melatonina }(1-3 \mathrm{mg}) \text { ao deitar } \\
\text { - Considerar a suspensão de antidepressivos serotoninérgicos, pelo risco de agravamento de RBD e } \\
\text { síndrome das pernas inquietas }\end{array}$ \\
\hline Alterações urinárias & $\begin{array}{l}\text { - Excluir infeção do trato urinário } \\
\text { - Noctúria: reduzir ingestão hídrica depois das } 18 \text { horas, aumentar dose noturna de levodopa, tratar } \\
\text { hipertrofia prostática } \\
\text { - Referenciar para Urologia: consoante o tipo de disfunção, poderá estar indicado cloreto de tróspio, } \\
\text { oxibutinina, tansulosina, toxina botulínica tipo A injetada no músculo detrusor da bexiga }\end{array}$ \\
\hline Obstipação & $\begin{array}{l}\text { - Reforço de ingestão hídrica, alimentos ricos em fibra e atividade física } \\
\text { - Laxantes com macrogol ou suplementos ricos em fibras }\end{array}$ \\
\hline Disfunção gástrica & $\begin{array}{l}\text { - Domperidona (30 - } 60 \mathrm{mg} / \mathrm{dia} \text {, dividida em } 3 \text { doses); metoclopramida deve ser evitada pelo risco de } \\
\text { agravamento do parkinsonismo }\end{array}$ \\
\hline Sialorreia & $\begin{array}{l}\text { - Atropina gotas } 1 \% \text { ( } 1 \text { - } 2 \text { gotas na cavidade oral, lateralmente e por baixo da língua, até } \\
4 \text { vezes/dia) } \\
\text { - Em casos graves toxina botulínica tipo A/B injetada nas parótidas (riscos devem ser devidamente } \\
\text { ponderados) }\end{array}$ \\
\hline Disfunção sexual & $\begin{array}{l}\text { - Descontinuar fármacos serotoninérgicos e bloqueadores alfa-adrenérgicos } \\
\text { - Se disfunção eréctil, sem contraindicações: inibidor da fosfodiesterase } 5 \text { (experiência é maior com } \\
\text { sildenafil } 50 \text { - } 100 \mathrm{mg} \text { ) } \\
\text { - Injeções subcutâneas de apomorfina ( } 5 \text { - } 10 \text { minutos antes da relação sexual) poderão ter } \\
\text { benefício, bem como injeções intracavernosas de alprostadil }\end{array}$ \\
\hline
\end{tabular}

diagnósticos é importante - cerca de $24 \%$ em centros especializados. ${ }^{48} \mathrm{~A}$ história farmacológica deve ser sempre revista, uma vez que a iatrogenia é a segunda causa mais frequente de parkinsonismo a motivar referenciação à consulta de Neurologia, com importantes implicações terapêuticas e prognósticas. Apesar da frequente associação aos fármacos antipsicóticos, sobretudo de primeira geração e alguns de segunda (e.g. haloperidol, clorpromazina, risperidona, olanzapina), outros fármacos como anti-eméticos (e.g metoclopramida), parassimpaticomiméticos (e.g. inibidores da acetilcolinesterase), antagonistas dos canais de cálcio (e.g. flunarizina, cinarizina, trimetazidina, 
verapamilo), anti-arrítmicos (e.g. amiodarona) e fármacos anti-epiléticos (e.g. ácido valpróico) podem estar também implicados. A tetrabenazina e o lítio estão também entre os fármacos que condicionam risco variável de parkinsonismo como efeito adverso. ${ }^{1,26,49,50}$

Esta problemática deve ser encarada com particular cuidado nos doentes idosos, frequentemente polimedicados e com maior risco de deterioração cognitiva e alteração comportamental. A simetria de sintomas é mais frequente no parkinsonismo iatrogénico (i.e. induzido por fármacos), existindo muitas vezes outros sintomas típicos de iatrogenia (e.g. tremor postural, acatísia, confusão mental). No entanto, dados mais recentes sugerem que numa proporção importante de casos (até $20 \%$ ), os sintomas podem ser igualmente assimétricos quando secundários a fármacos. Uma pista pode ser o facto de os sintomas serem habitualmente dose-dependentes e reversíveis até três meses após a suspensão farmacológica. Da história clínica, a hiposmia foi apontada como fator diferenciador a favor de DP versus iatrogenia. ${ }^{49}$ Por fim, a marcação com rádio-fármacos de transportadores dopaminérgicos pré-sinápticos no estriado é normal na iatrogenia, em oposição às causas neurodegenerativas. ${ }^{50}$ A designação de 'parkinsonismo vascular' é frequentemente atribuída a sintomas de parkinsonismo com sintomas predominantes nos membros inferiores, com alteração da marcha e declínio cognitivo em doentes com alterações de substância branca na imagem cerebral, devido a doença vascular de pequenos vasos crónica. No entanto, a ausência de um marcador clínico ou imagiológico para esta entidade e a fraca correlação entre a imagem e a clínica têm sido objeto de grande debate, pelo que este diagnóstico se reveste de elevada controvérsia. ${ }^{51}$ De entre as causas mais comuns de parkinsonismo degenerativo estão a demência com corpos de Lewy, a AMS, a PSP, a degenerescência córtico-basal e a síndrome de tremor e ataxia ligada ao $\mathrm{X}$ frágil (fragile-X tremor ataxia syndrome, FXTAS). ${ }^{19}$

\section{Terapêutica}

O tratamento da DP deve ser individualizado e pode envolver a necessidade de uma equipa multidisciplinar. Não existem atualmente terapêuticas capazes de diminuir ou travar o processo neurodegenerativo (modificadoras do curso da doença), nem de substituir os neurónios perdidos (neuro-restauradoras). Com o recente surgimento de terapêuticas imunomoduladoras e baseadas em oligonucleotídeos, existe um interesse generalizado no potencial de futuras terapêuticas tendo como alvo a alfa-sinucleína. ${ }^{52}$ Contudo, permanecem importantes questões por responder, tais como a fase ideal para a sua introdução e a população exata a ser selecionada para os ensaios clínicos, tarefa que poderia ser facilitada pela identificação de biomarcadores da doença.

As intervenções terapêuticas atualmente disponíveis apenas têm a capacidade de melhorar os sintomas da doença, com eficácia notoriamente mais elevada nos sintomas motores. A levodopa foi o primeiro fármaco usado eficazmente para o tratamento da DP e, mais de 50 anos após a sua introdução, permanece o mais eficaz no tratamento dos sintomas motores. ${ }^{53}$ No entanto, apesar de estudos iniciais terem sugerido um papel 'modificador da doença' da levodopa, só muito recentemente um estudo aleatorizado veio acrescentar evidência sólida no sentido de que o benefício da levodopa se remete apenas para o tratamento sintomático da doença. ${ }^{54}$ Por tudo isto, a decisão de iniciar ou não terapêutica deve ser guiada pelos sintomas e contexto clínico do doente, devendo ser sempre discutida com este.

Durante anos, temia-se que a levodopa se associasse ao aparecimento de complicações motoras mais precocemente no curso da doença, como deterioração de fim de dose (wearing-off) e discinésias de pico de dose, podendo estas aparecer mesmo numa fase inicial, tendo um carácter imprevisível. ${ }^{1,55} \mathrm{~A}$ interpretação da evidência científica favorecia iniciar o tratamento com agonistas dopaminérgicos. Esta classe farmacológica pode causar efeitos adversos importantes, como edema periférico, sonolência diurna e perturbações do controlo de impulsos (compras compulsivas, jogo patológico, hiperfagia, hipersexualidade e comportamentos motores repetitivos sem propósito ou punding), que condicionam taxas de descontinuação da terapêutica mais elevadas do que nos pacientes em tratamento com levodopa. Por outro lado, a evidência mais recente confirma a ideia de que as complicações motoras surgem independentemente do momento de início da levodopa. ${ }^{56,57}$ Assim, os doentes não devem ser privados de levodopa, quando esta é a melhor terapêutica sintomática no caso em questão, o que acontece em particular nos pacientes mais idosos, com maior risco de deterioração cognitiva, múltiplas co-morbilidades e medicações que podem não ser candidatos a agonista dopaminérgico; ou ainda naqueles que tiveram benefício sintomático sub-ótimo com as outras terapêuticas já testadas. A Tabela 2 detaIha as opções terapêuticas disponíveis para tratamento dos sintomas motores da DP.

Uma explicação exaustiva sobre o tratamento das complicações motoras ultrapassa o âmbito deste artigo, pelo que deixamos aqui apenas breves considerações. A duração e estadio da doença, a exposição à levodopa e a dose cumulativa (sobretudo doses superiores a $600 \mathrm{mg} / \mathrm{dia}$ ), a motilidade gástrica errática, doença em idade jovem, género feminino e o baixo peso corporal parecem condicionar maior risco. O fracionamento das doses, a melhoria da absorção (antecipando a toma em pelo menos 30 minutos antes das refeições) e a associação com um inibidor da descarboxílase periférica (benserazida ou carbidopa - o que já sucede nas actuais formulações disponíveis) são estratégias disponíveis para melhorar a farmacocinética da levodopa. As potenciais estratégias em doentes com complicações motoras encontram-se resumidas na Tabela 3 e, em mais detalhe, numa revisão recente. ${ }^{58}$

Nos doentes com DP, boa resposta à levodopa e com complicações motoras após otimização da terapêutica por neurologista, ou com efeitos adversos intoleráveis da medicação, a DBS (do núcleo subtalâmico ou globo pálido 
Tabela 2 - Principais terapêuticas farmacológicas disponíveis para o tratamento da doença de Parkinson em Portugal, para além da levodopa

\begin{tabular}{|c|c|}
\hline Classe farmacológica & Fármacos disponíveis \\
\hline $\begin{array}{l}\text { Agonistas dopaminérgicos } \\
\text { não-ergotamínicos }\end{array}$ & $\begin{array}{l}\text { Ropinirole libertação imediata }(0,25 \mathrm{mg} \text { a } 5 \mathrm{mg} \text {, TID) } \\
\text { Ropinirole libertação prolongada (2 mg a } 8 \mathrm{mg}, \mathrm{QD}) \\
\text { Pramipexole libertação imediata }(0,088 \mathrm{mg} \text { a } 0,7 \mathrm{mg} \text {, TID) } \\
\text { Pramipexole libertação prolongada }(0,26 \mathrm{mg} \text { a } 3,15 \mathrm{mg}, \mathrm{QD}) \\
\text { Rotigotina }(2,4,6,8 \mathrm{mg} / 24 \mathrm{~h} \text {, adesivo transdérmico) } \\
\text { Apomorfina ( } 10 \mathrm{mg} / \mathrm{mL} \text {, injeção subcutânea ou perfusão contínua) }\end{array}$ \\
\hline $\begin{array}{l}\text { Inibidores da monoamina } \\
\text { oxidase B (MAO-B) }\end{array}$ & $\begin{array}{l}\text { Selegilina ( } 5 \mathrm{mg}, \mathrm{BID}) \\
\text { Selegilina orodispersível (1,25 mg, QD) } \\
\text { Rasagilina (1 mg, QD) } \\
\text { Safinamida ( } 50 \text { a } 100 \mathrm{mg}, \mathrm{QD})\end{array}$ \\
\hline $\begin{array}{l}\text { Inibidores da catecol-o- } \\
\text { metiltransferase (COMT) }\end{array}$ & $\begin{array}{l}\text { Entacapone ( } 200 \mathrm{mg} \text {, em associação com levodopa/carbidopa ou formulação isolada, TID a QID) } \\
\text { Opicapone (50 mg, QD) }\end{array}$ \\
\hline Anticolinérgicos* & $\begin{array}{l}\text { Trihexifenidilo (2 a } 5 \mathrm{mg} \text {, TID a QID) } \\
\text { Cloridrato de biperideno (2 a } 4 \text { mg, QD a QID) }\end{array}$ \\
\hline Outros & Amantadina (100 mg, BID a TID) \\
\hline
\end{tabular}

*perfil de segurança adverso pelo risco de deterioração mnésica, confusão, psicose, retenção urinária, xerostomia, xeroftalmia e precipitação de crises de glaucoma, pelo que o seu uso deve ser limitado a casos seleccionados de pacientes jovens (<60 anos), cognitivamente íntegros, com tremor incapacitante e refratário às outras medicações anti-parkinsónicas.

LP: libertação prolongada; QD: uma toma/dia; BID: duas tomas/dia; TID: três tomas/dia; QID: quatro tomas/dia

interno) é uma opção terapêutica eficaz e segura para pacientes cuidadosamente selecionados, sem contra-indicações para esta cirurgia. ${ }^{59,60}$ Desde 2002, ano em que o programa de DBS foi iniciado em Portugal, no Hospital de São João, realizaram-se no nosso país centenas destas intervenções. A referenciação dos doentes pode ser feita para um centro especializado, aconselhando-se que a mesma seja realizada pelo Neurologista do doente. A apomorfina subcutânea é uma alternativa a considerar nestes doentes, incluindo nos que têm contra-indicação cirúrgica ou que recusam cirurgia, estando atualmente já disponível em Portugal sob a forma de caneta ou ampola para perfusão contínua. ${ }^{61} \mathrm{~A}$ levodopa por infusão intestinal em gel é outra possibilidade para doentes com flutuações motoras. ${ }^{62}$ Apesar de todas estas estratégias, os bloqueios (freezing) da marcha e a instabilidade postural são sintomas muitas vezes refratários às terapêuticas atualmente disponíveis.

Nunca é demais enfatizar o papel positivo do reforço da capacidade física dos doentes, através de treino de marcha, equilíbrio e reforço muscular. ${ }^{63}$ Estão publicados dezenas de estudos aleatorizados cujas intervenções são centradas em exercício físico e/ou fisioterapia. No entanto, a evidência que suporta estas terapêuticas permanece globalmente inferior à dos fármacos e opções cirúrgicas disponíveis para o tratamento dos sintomas da DP. ${ }^{64}$

\section{CONCLUSÃO}

Apesar dos marcadores patológicos da doença serem bem conhecidos, nomeadamente a deposição progressiva de alfa-sinucleína no sistema nervoso central, a fisiopatologia da DP está longe de estar esclarecida, em parte porque

Tabela 3 - Estratégias terapêuticas nas complicações motoras da doença de Parkinson. A gestão clínica destas situações é particularmente complexa e deve ser feita por neurologista

\begin{tabular}{ll}
\hline Complicação motora & Opçães terapêuticas \\
\hline $\begin{array}{l}\text { Wearing-off ou } \\
\text { deterioração de fim } \\
\text { de dose }\end{array}$ & - Adicionar um inibidor da MAO-B \\
& - Adicionar um inibidor da COMT \\
& - Aumentar a dose de levodopa em cada toma/diminuir o intervalo entre tomas de levodopa \\
- Acinésia matinal ou & - Adicionar um inibidor da COMT \\
noturna & Adicionar/aumentar a dose noturna de agonista dopaminérgico oral ou introduzir/subir a dose \\
& - Adicionar levodopa de libertação prolongada à noite \\
& - Estimulação cerebral profunda \\
& Diminuir a dose de levodopa em cada toma/aumentar o intervalo de tempo entre tomas \\
Discinésias & - Adicionar amantadina \\
& - Diminuir a dose/parar agonista dopaminérgico \\
Flutuações motoras & Pstimulação cerebral profunda \\
complexas & - Gestão por especialista em doenças do movimento/doença de Parkinson sempre que possível \\
\hline
\end{tabular}


múltiplas vias estão envolvidas no processo neurodegenerativo. Atualmente ainda não dispomos de um teste ou biomarcador que confirme o diagnóstico de DP, pelo que este é clínico, apoiado por investigação complementar adequada. Recentemente foram propostos novos critérios de diagnóstico, enfatizando critérios de exclusão e red flags para o diagnóstico, sendo fundamentais a história clínica e o exame físico para a destrinça entre DP e os seus principais diagnósticos diferenciais, nomeadamente causas secundárias como o parkinsonismo iatrogénico. Após a introdução da levodopa, a DBS surgiu como uma intervenção de grande impacto sintomático no tratamento da DP, mas está indicada apenas numa minoria de doentes, tendo em conta os critérios de inclusão e exclusão. Uma proporção importante da incapacidade na DP é atribuível às complicações motoras, aos sintomas axiais (menos responsivos à levodopa), e aos sintomas não-motores. Paralelamente, temos assistido a uma expansão das opções terapêuticas, nomeadamente dirigidas às complicações motoras da doença. No entanto, as intervenções terapêuticas atualmente disponíveis para a DP estão longe de satisfazer as necessidades clínicas, pelo que se aguardam com expectativa os resultados das múltiplas linhas de investigação em curso.

\section{CONFLITOS DE INTERESSE}

Verónica Cabreira recebeu suporte financeiro por parte de Roche para assistir a reuniões científicas. João Massano recebeu honorários de consultoria de Bial, Merck Sharp \& Dohme, e Zambon; recebeu suporte financeiro por parte de Bial, Boston Scientific, GE Healthcare, Grunenthal, Medtronic, e Novartis para fazer palestras ou assistir a reuniões científicas.

\section{FONTES DE FINANCIAMENTO}

Este trabalho não recebeu qualquer tipo de suporte financeiro de nenhuma entidade no domínio público ou privado.

\section{REFERÊNCIAS}

1. Lees AJ, Hardy J, Revez T. Parkinson's disease. Lancet. 2009; 373:2055-66.

2. Kempster PA, Hurwitz B, Lees AJ. A new look at James Parkinson's Essay on the Shaking Palsy. Neurology. 2007;69:482-85.

3. Goetz CG. The history of Parkinson's disease: early clinical descriptions and neurological therapies. Cold Spring Harb Perspect Med. 2011;1:a008862

4. Morrish PK, Sawle GV, Brooks DJ. An [18F]dopa-PET and clinical study of the rate of progression in Parkinson's disease. Brain. 1996;119:585-91.

5. Braak H, Del Tredici K, Rüb U, De Vos RA, Jansen Steur EN, Braak E. Staging of brain pathology related to sporadic Parkinson's disease. Neurobiol Aging. 2003;24:197-211.

6. Savica R, Bradley BF, Mielke MM. When do a-Synucleinopathies start? An epidemiological timeline a review. JAMA Neurol. 2018;75:503-09.

7. Ferreira JJ, Gonçalves N, Valadas A, Januário C, Silva MR, Nogueira L, et al. Prevalence of Parkinson's disease: a population-based study in Portugal. Eur J Neurol. 2017;24:748-50.

8. Kaasinen V, Vahlberg $\mathrm{T}$, Suominen S. Increasing age-adjusted male-to-female incidence ratio of Parkinson's disease. Mov Disord. 2015;30:286-8.

9. Dorsey ER, Elbaz A, Nichols E, Abd-Allah F, Abdelalim A, Adsuar JC, et al. Global, regional, and national burden of Parkinson's disease, 1990-2016: a systematic analysis for the Global Burden of Disease Study 2016. Lancet Neurol. 2018;17:939-53

10. Hirsch L, Jette N, Frolkis A, Steeves T, Pringsheim T. The Incidence of Parkinson's disease: a systematic review and meta-analysis. Neuroepidemiology. 2016;46:292-300.

11. De Pablo-Fernandez E, Tur C, Revesz T, Lees AJ, Holton JL, Warner TT. Association of autonomic dysfunction with disease progression and survival in Parkinson disease. JAMA Neurol. 2017;74:970-76.

12. Pringsheim T, Jette N, Frolkis A, Steeves TD, Macleod AD, Taylor KS, et al. Mortality in Parkinson's disease: a systematic review and metaanalysis. Mov Disord. 2014;29:1615-22.

13. Macleod AD, Dalen I, Tysnes OB, Larsen JP, Counsell CE. Development and validation of prognostic survival models in newly diagnosed Parkinson's disease. Mov Disord. 2018;33:108-16.

14. Jankovic J, McDermott M, Carter J, Gauthier S, Goetz C, Golbe L, et al. Variable expression of Parkinson's disease: a base-line analysis of the DATATOP cohort. The Parkinson Study Group. Neurology. 1990;40:1529-34

15. Leehey M, Luo S, Sharma S, Wills AM, Bainbridge JL, Wong PS, et al. Association of metabolic syndrome and change in Unified Parkinson's Disease Rating Scale scores. Neurology. 2017;89:1789-94.

16. Wills AM, Pérez A, Wang J, Su X, Morgan J, Rajan SS, et al. Association between change in body mass index, inified Parkinson's disease rating scale scores, and survival among persons with Parkinson disease. JAMA Neurol. 2016;73:321-8. relationship? Mov Disord. 2018;33:702-03.

18. Houser MC, Chang J, Factor SA, Molho ES, Zabetian CP, Hill-Burns EM, et al. Stool immune profiles evince gastrointestinal inflammation in Parkinson's disease. Mov Disord. 2018;33:793-804.

19. Massano J, Bhatia KP. Clinical approach to Parkinson's disease: features, diagnosis, and principles of management. Cold Spring Harb Perspect Med. 2012;2:a008870.

20. Domingo A, Klein C. Genetics of Parkinson disease. Handb Clin Neurol. 2018;147:211-27.

21. Ferreira M, Massano J. An updated review of Parkinson's disease genetics and clinicopathological correlations. Acta Neurol Scand 2017;135:273-84

22. Sidransky E, Lopez $\mathrm{G}$. The link between the GBA gene and parkinsonism Lancet Neurol. 2012;11:986-98.

23. O'Regan G, Desouza RM, Balestrino R, Schapira $A H$. Glucocerebrosidase mutations in Parkinson disease. J Parkinsons Dis. 2017;7:411-22

24. Goldman JG, Andrews H, Amara A, Naito A, Alcalay RN, Shaw LM, et al. Cerebrospinal fluid, plasma, and saliva in the BioFIND study: relationships among biomarkers and Parkinson's disease features. Mov Disord. 2018;33:282-88.

25. Ortuño-Lizarán I, Beach TG, Serrano GE, Walker DG, Adler $\mathrm{CH}$, Cuenca N. Phosphorylated $\alpha$-synuclein in the retina is a biomarker of Parkinson's disease pathology severity. Mov Disord. 2018;3:e001126.

26. Massano J. Doença de Parkinson: actualização clínica. Acta Med Port. 2011;24:827-34.

27. Gibb WR, Lees AJ. The relevance of the Lewy body to the pathogenesis of idiopathic Parkinson's disease. J Neurol Neurosurg Psychiatry. 1988:51:745-52.

28. Postuma RB, Berg D, Stern M, Poewe W, Olanow CW, Oertel W, et al. MDS clinical diagnostic criteria for Parkinson's disease. Mov Disord. 2015;30:1591-601.

29. Kägi G, Bhatia KP, Tolosa E. The role of DAT-SPECT in movement disorders. J Neurol Neurosurg Psychiatry. 2010;81:5-12.

30. Bajaj N, Hauser RA, Grachev ID. Clinical utility of dopamine transporter single photon emission CT (DaT-SPECT) with (123I) ioflupane in diagnosis of parkinsonian syndromes. J Neurol Neurosurg. Psychiatry. 2013;84:1288-95.

31. Stoessl AJ. Neuroimaging in Parkinson's disease: from pathology to diagnosis. Parkinsonism Relat Disord. 2012;8:72-81.

32. Ishibashi K, Saito Y, Murayama S, Kanemaru K, Oda K, Ishiwata K, et al. Validation of cardiac123I-MIBG scintigraphy in patients with Parkinson's disease who were diagnosed with dopamine PET. Eur J Nucl Med Mol Imaging. 2010;37:3-11.

33. Pilotto A, Yilmaz R, Berg D. Developments in the role of transcranial sonography for the differential diagnosis of parkinsonism. Curr Neurol Neurosci Rep. 2015;15:43. 
34. Berardelli A, Wenning GK, Antonini A, Berg D, Bloem BR, Bonifati V, et al. EFNS/MDS-ES/ENS [corrected] recommendations for the diagnosis of Parkinson's disease. Eur J Neurol. 2013;20:16-34.

35. Rocha H, Cerejo A, Garrett MC, Massano J. Reversible parkinsonism due to a large intracranial tumour. BMJ Case Rep. 2012;2012:bcr2012007823

36. Mahlknecht P, Hotter A, Hussl A, Esterhammer R, Schocke M, Seppi K. Significance of MRI in diagnosis and differential diagnosis of Parkinson's disease. Neurodegener Dis. 2010;7:300-18.

37. Cabreira V, Brandão $\mathrm{E}$, Massano J. More than meets the eye: an unusual Parkinson's disease mimic. Int J Clin Neurosci Ment Health. 2018;5:8.

38. Carvalho V, Massano J. Tremor: a clinical guide for the non-neurologist. Acta Med Port. 2019;32:149-57.

39. Morris ME, lansek R, Matyas TA, Summers JJ. Ability to modulate walking cadence remains intact in Parkinson's disease. J Neurol Neurosurg Psychiatry. 1994;57:1532-4.

40. Khan NL, Brooks DJ, Pavese N, Sweeney MG, Wood NW, Lees AJ, et al. Progression of nigrostriatal dysfunction in a parkin kindred: an [18F]dopa PET and clinical study. Brain. 2002;125:2248-56.

41. Poewe W. Non-motor symptoms in Parkinson's disease. Eur J Neurol. 2008;151:S14-20.

42. Hely MA, Reid WG, Adena MA, Halliday GM, Morris JG. The Sydney Multicenter Study of Parkinson's disease: the inevitability of dementia at 20 years. Mov Disord. 2008;23:837-44.

43. Litvan I, Goldman JG, Tröster Al, Schmand BA, Weintraub D, Petersen RC, et al. Diagnostic criteria for mild cognitive impairment in Parkinson's disease: Movement Disorder Society Task Force guidelines. Mov Disord. 2012;27:349-56.

44. Dalrymple-Alford JC, MacAskill MR, Nakas CT, Livingston L, Graham C, Crucian GP, et al. The MoCA: well-suited screen for cognitive impairment in Parkinson disease. Neurology. 2010;75:1717-25.

45. Schapira AH, Tolosa E. Molecular and clinical prodrome of Parkinson disease: Implications for treatment. Nat Rev Neurol. 2010;6:309-17.

46. Martínez-Fernández R, Schmitt E, Martinez-Martin P, Krack P. The hidden sister of motor fluctuations in Parkinson's disease: a review on nonmotor fluctuations. Mov Disord. 2016;31:1080-94.

47. Baig F, Lawton M, Rolinski M, Ruffmann C, Nithi K, Evetts SG, et al. Delineating nonmotor symptoms in early Parkinson's disease and firstdegree relatives. Mov Disord. 2015;30:1759-66.

48. Jankovic J. Parkinson's disease: clinical features and diagnosis. Neurol Neurosurg Psychiatry. 2008;79:368-76.

49. Ward KM, Citrome L. Antipsychotic-related movement disorders: druginduced Parkinsonism vs. tardive dyskinesia - key differences in pathophysiology and clinical management. Neurol Ther. 2018;7:233-48.

50. Brigo F, Erro R, Marangi A, Bhatia K, Tinazzi M. Differentiating druginduced parkinsonism from Parkinson's disease: An update on non-motor symptoms and investigations. Park Relat Disord. 2014;20:808-14.51.

51. Vizcarra JA, Lang AE, Sethi KD, Espay AJ. Vascular Parkinsonism: deconstructing a syndrome. Mov Disord. 2015;30:886-94.

52. Lang AE, Espay AJ. Disease modification in Parkinson's disease: current approaches, challenges, and future considerations. Mov Disord. 2018;33:660-77.

53. Gray R, Ives N, Rick C, Patel S, Gray A, Jenkinson C, et al. Long term effectiveness of dopamine agonists and monoamine oxidase $B$ inhibitors compared with levodopa as initial treatment for Parkinson's disease (PD MED): a large, open-label, pragmatic randomised trial. Lancet. 2014;384:1196-205.

54. Verschuur CV, Suwijn SR, Boel JA, Post B, Bloem BR, van Hilten JJ, et al. Randomized delayed-start trial of levodopa in Parkinson's disease. N Engl J Med. 2019;380:315-34.

55. Stocchi F, Jenner P, Obeso JA. When do levodopa motor fluctuations first appear in Parkinson's disease? Eur Neurol. 2010;63:257-66.

56. Olanow CW, Stocchi F. Levodopa: a new look at an old friend. Mov Disord. 2018;33:859-66.

57. Fox SH, Lang AE. "Don't delay, start today": delaying levodopa does not delay motor complications. Brain. 2014;33:859-66.

58. Cabreira V, Soares-da-Silva P, Massano J. Contemporary options for the management of motor complications in Parkinson's disease: updated clinical review. Drugs. 2019;79:593-608.

59. Okun MS, Gallo BV, Mandybur G, Jagid J, Foote KD, Revilla FJ, et al. Subthalamic deep brain stimulation with a constant-current device in Parkinson's disease: An open-label randomised controlled trial. Lancet Neurol. 2012;11:140-9.

60. Weaver FM, Follett KA, Stern M, Harris CL, Rothlind J, Lai EC, et al. Randomized trial of deep brain stimulation for Parkinson disease: thirtysix-month outcomes. Neurology. 2012;79:55-65.

61. Katzenschlager R, Poewe W, Rascol O, Trenkwalder C, Deuschl G, Chaudhuri KR, et al. Apomorphine subcutaneous infusion in patients with Parkinson's disease with persistent motor fluctuations (TOLEDO): a multicentre, double-blind, randomised, placebo-controlled trial. Lancet Neurol. 2018;17:749-59.

62. Antonini A, Fung VS, Boyd JT, Slevin JT, Hall C, Chatamra K, et al. Effect of levodopa-carbidopa intestinal gel on dyskinesia in advanced Parkinson's disease patients. Mov Disord. 2016;31:530-7.

63. Keus SH, Bloem BR, Hendriks EJ, Bredero-Cohen AB, Munneke M. Evidence-based analysis of physical therapy in Parkinson's disease with recommendations for practice and research. Mov. Disord. 2007;22:451-60.

64. Fox SH, Katzenschlager R, Lim SY, Barton B, de Bie RM, Seppi K, et al. International Parkinson and movement disorder society evidence-based medicine review: Update on treatments for the motor symptoms of Parkinson's disease. Mov Disord. 2018;33:1248-66. 